\title{
Überaktive Blase
}

\section{„Die Miktion organisieren können“}

- Nach Auswertung von 82 Studien mit Evidenzlevel 1 können moderne Präparate wie Solifenacin beispielsweise Drang- und Dranginkontinenzepisoden wirksam reduzieren - so das Ergebnis einer Recherche von Cornu et al (Progrès en Urologie 2013;23(4):227-236).

Nach Definition der International Continence Society (ICS) gilt der imperative Harndrang als Leitsymptom der Erkrankung. Er ist zudem Auslöser für die Symptomtrias Nykturie, Pollakisurie und einer möglichen Inkontinenz. „,Die Patienten fühlen sich vom überfallartigen Harndrang wegen seiner unvorhersehbaren Wirkung auf Alltagssituationen stark beeinträchtigt", erklärt PD Dr. Maximilian Burger im Gespräch mit der Ärzte Zeitung. „Die Drangepisoden lassen den Patienten oft keine Zeit, ihre Blasenentleerung zu orga- nisieren." Sie kommen nicht rechtzeitig zur Toilette, sodass der imperative Harndrang zur spontanen Blasenentleerung - also zur Inkontinenz - führt.

\section{Therapieziel Harndrang}

In mehreren Studien mit dem modernen Anticholinergikum Solifenacin hat man den Harndrang als primären Endpunkt gewählt: In der SUNRISE1-Studie, einer 16-wöchigen, randomisierten MulticenterStudie mit knapp 900 Patienten, verwendeten Cardozo et al. die PPIUS-Bewertungsskala zur Beurteilung des Harndrangs. Die Skala reicht von 0 (kein Harndrang) bis 4 (starker Harndrang). In der Untersuchung senkte Solifenacin die Anzahl der schweren Harndrang-Epidoden um -2,6 im Vergleich zu -1,8 unter Placebo [Cardozo L et al. BJU International 2008;

\section{Ginkgo-Extrakt bei chronischem Tinnitus Synergistische Effekte nutzen}

- Patienten mit chronischem Tinnitus assoziieren das Phänomen mit negativen Emotionen und fokussieren ihre Aufmerksamkeit auf das Ohrgeräusch. Mit steigender psychischer Belastung und abnehmender Stresstoleranz wird der Tinnitus in der Folge noch stärker wahrgenommen, erläuterte Prof. Dr. Matthias Tisch, Bundeswehrkrankenhaus Ulm. Deshalb sei es in der Therapie mit durchblutungsfördernden Maßnahmen allein nicht getan.

\section{Therapie ruht auf zwei Stützpfeilern}

Die Arbeitsgemeinschaft der Wissenschaftlichen Medizinischen Fachgesellschaften (AWMF) empfiehlt beim Tinnitus eine rheologische Akuttherapie mit Hämodilution zur Verbesserung der Fluidität und Senkung der Plasmaviskosität. Eine solche Therapie z.B. mit Kortison oder Hydroxyethylstärke ist aber laut Tisch mit erheblichen Nebenwirkungen assoziiert und kommt für zahlreiche Patienten nicht in- frage. Anders sieht es beim Extrakt aus Ginkgo biloba aus, dessen viskositätssenkende Wirkung belegt ist, ohne dass eine relevante Veränderung von Gerinnungsparametern in Kauf zu nehmen ist.

Der Ginkgo-Extrakt EGb 761 ${ }^{\circledR}$ (Tebo$\mathrm{nin}^{\circledR}$ ) wurde in mehreren Tinnitus-Studien erfolgreich geprüft. So bei gut 100 Patienten mit akutem bis subakutem Tinnitus: Rund $50 \%$ erfuhren unter der Behandlung eine teilweise stark ausgeprägte Verbesserung, berichtete Tisch. Auch bei Patienten, die bereits im Schnitt 4,5 Jahre an Tinnitus litten, konnte im Vergleich zu Placebo innerhalb von zwölf Wochen eine signifikante Abnahme der störenden Ohrgeräusche durch EGb $761^{\circledR}$ erreicht werden $(p<0,04)$.

Leitliniengemäß kann Ginkgo-Extrakt damit laut Tisch bei akutem Tinnitus zur Verringerung der Viskosität zum Einsatz kommen, durchaus auch in Kombination mit weiteren rheologischen Maßnahmen.
102:1120-1127, http://www.ncbi.nlm.nih gov/pubmed/18990175].

In der multizentrischen, randomisierten placebokontrollierten VENUS-Studie sank die Anzahl der täglichen Drangepisoden unter Solifenacin um durchschnittlich $-3,9$ im Vergleich zu -2,7 unter Placebo. Gleichzeitig konnte die Vorwarnzeit (Verspüren des ersten Harndrangempfindens bis zur Miktion) um 31,5 Sekunden gesteigert werden [Karram MM et al. Urology 2009; 73:1418, http://www.ncbi.nIm.nih.gov/pubmed/18995887]. „Durch die längert Vorwarnzeit und die selteneren Drangepisoden gelingt es den Patienten, geordnet die Toilette aufzusuchen", so Burger. Das mache für sie einen wichtigen Unterschied.

- Dr. Constanze Löffler

Quelle: Interview mit PD Dr. med. Maximilian Burger durch die Ärzte Zeitung

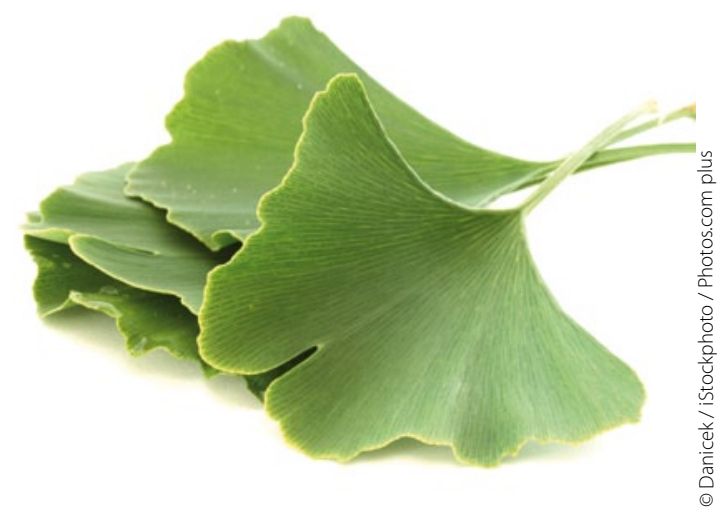

Die Kraft der Gingkoblätter nutzen ...

Zusätzlich könne die frühe Gabe des Extrakts Umlernprozesse zur Rückbildung der fehlerhaften Hörverarbeitung fördern. In der chronischen Phase muss man laut Tisch in erster Linie auf Retraining-Strategien setzen und die synergistischen Effekte des Ginkgo-Spezialextrakts bei der Förderung der neuronalen Plastizität im auditorischen Cortex nützen.

- Dr. Katharina Arnheim

Quelle: Symposium „Tinnitus - ,Phantomschmerz' der Cochlea. Moderne Konzepte zur Tinnitusentstehung und -therapie" im Rahmen der DGIM-Jahrestagung 2013, Wiesbaden, April 2013 (Veranstalter: Dr. Willmar Schwabe) 